\title{
Aplicación del permanganato potásico para la eliminación de cianuros de cobre en aguas residuales de la planta de lixiviación en una mina de oro (II): Ensayos en planta piloto(*)
}

\author{
J. P. Sancho*, B. Fernández*, J. Ayala*, M. P. García* y A. Lavandeira**
}

Resumen

\begin{abstract}
La búsqueda de un tratamiento que permita la detoxificación de las aguas residuales generadas durante los procesos industriales, ha sido una constante para todas las empresas en general y para las explotaciones mineras auríferas en particular, cuyas aguas residuales suelen contener elevadas concentraciones de compuestos cianurados con una elevada toxicidad. En el trabajo de investigación previo, desarrollado en el laboratorio, se demostró la eficacia del permanganato potásico como agente oxidante de aguas residuales cianuradas, procedentes de una planta hidrometalúrgica de oro, consiguiendo la destrucción de los complejos cianurados de cobre presentes en solución y la posterior eliminación del metal por precipitación como hidróxido. En este trabajo se presentan las conclusiones obtenidas tras la implantación del proceso desarrollado en el laboratorio, a escala de planta piloto.
\end{abstract}

\section{Uses of the potassium permanganate to eliminate copper cyanide from waste water resulting from a lixiviation plant in a gold mine (II): Pilot plant experiences}

Abstract

Keywords
The search for a detoxification treatment of the wastewater generated during industrial processes, has been a constant for all companies in general and for gold mining in particular, whose wastewater generally contains high concentrations of cyanide compounds with high toxicity. In the previous research work, developed in the laboratory, the efficacy of potassium permanganate as an oxidizing agent for cyanidic wastewater, from a gold hydrometallurgical plant, has been demonstrated, achieving the destruction of copper cyanide complexes present in solution and the subsequent metal removal by precipitation as hydroxide. This paper presents the conclusions obtained after the implementation of the process developed in the laboratory, at pilot-plant scale.

\section{INTRODUCCIÓN}

Los cianuros alcalinos se utilizan en la extracción de metales preciosos de las menas auríferas a escala mundial, desde que su uso fue patentado ${ }^{[1]}$. A pesar de los problemas ambientales que presenta éste y de la gran cantidad de investigaciones sobre otros procesos de disolución menos contaminantes, actualmente se sigue utilizando ampliamente debido a su bajo costo y a la simplicidad del proceso ${ }^{[2}$ y 3$]$.

La complejidad de muchas de las menas tratadas hace que se produzca un consumo importante de cianuro en el proceso, debido a reacciones secundarias con otros metales como hierro, níquel, plata, zinc, y cobre, preferentemente, formándose cianuros, cianatos, y también tiocianatos por reacción con los sulfuros presentes en la mena. En concreto, el cobre es considerado uno de los metales más problemáticos para el proceso de extracción por su elevada avidez por el cianuro y al hecho de que está presente en casi todas las menas auríferas y en particular en los yacimientos de Belmonte de Miranda (Asturias) ${ }^{[4 \text { y } 5]}$.

Todos estos compuestos mencionados relacionados con el cianuro, incluido el cianuro libre, son solubles y poseen un alto grado de contaminación, siendo necesaria la aplicación de procedimientos de deto-

(•) Trabajo recibido el día 20 de Mayo 2010 y aceptado en su forma final el día 25 de Enero de 2011.
* E.T.S.I.M.O. Independencia, 13 Oviedo, España.

** Río Narcea Gold Mines, S.A. 
xificación a las aguas de las plantas de tratamiento mineras, antes de ser vertidas a cauces públicos ${ }^{[6 \text { y } 7]}$.

Los principales métodos empleados: Degradación natural, Oxidación química, Precipitación y Biodegradación, presentan distintos niveles de efectividad, aunque ninguno de ellos por sí solo permite alcanzar el $100 \%$ de eliminación del contaminante, tal y como se apuntaba en el trabajo anterior ${ }^{[8-10]}$. Por otra parte, aquellos procesos que permiten obtener los mejores resultados, como es el caso del proceso de destrucción del cianuro con $\mathrm{SO}_{2}$, implican un elevado coste económico para las empresas que los implantan, tanto en inversiones como en el coste de operación, siendo necesario en ocasiones sobredimensionar las instalaciones para alcanzar los límites de destrucción requeridos. Por todo esto, parece conveniente la investigación y el desarrollo de nuevas alternativas ${ }^{[11-15]}$.

Los ensayos previos realizados en el estudio a nivel de laboratorio ${ }^{[16]}$ han demostrado la eficacia del permanganato potásico como oxidante de compuestos cianurados de cobre. En dicho estudio se desarrolló, a ese nivel, un proceso de detoxificación de aguas cianuradas de mina en el que se demuestra la versatilidad y eficacia del proceso desarrollado, obteniéndose elevadas tasas de reducción de cianuros, incluso para concentraciones extremas y con tiempos de reacción cortos.

Tras la valoración de los resultados del proceso anteriormente señalado, se planteó el posible cambio de escala del mismo hasta nivel industrial. Con este propósito se diseñó y construyó una pequeña planta piloto en las instalaciones de una empresa minero-metalúrgica asturiana. A pesar de algunas dificultades propias del diseño y construcción de la instalación, así como del hecho de trasladar un proceso realizado hasta el momento en condiciones controladas en el laboratorio a un proceso en continuo con todas las posibles variables que esto conlleva, se consiguió llevar a cabo la experiencia sin grandes problemas y con éxito final.
En este trabajo se presentan los resultados obtenidos en dicha experiencia poniéndose de manifiesto, la viabilidad del proceso a escala industrial.

\section{MATERIALES Y MÉTODOS DE MEDIDA}

\subsection{Aguas Residuales}

El agua tratada procedía del Agua de Proceso (A.P.) de la planta de tratamiento "El Valle" que la empresa Río Narcea Gold Mines, S.A. poseía en el concejo de Belmonte de Miranda (Asturias) y que actualmente pertenece a la empresa multinacional canadiense Orvana Minerals Corporation.

Durante el proceso de cianuración CIL (Carbon In Leach), que la empresa aplicaba para la disolución del oro de granulometría muy fina en la mena, siguiendo la ecuación (1) de Elsner (1846):

$$
\begin{gathered}
4 \mathrm{Au}+8 \mathrm{Na}(\mathrm{CN})+\mathrm{O}_{2}+2 \mathrm{H}_{2} \mathrm{O} \\
\Rightarrow 4 \mathrm{NaAu}(\mathrm{CN})_{2}+4 \mathrm{NaOH} \\
\mathrm{DG}^{\circ}=-233 \mathrm{~kJ}
\end{gathered}
$$

se generaba una pulpa residual que era enviada a un tanque de detoxificación con anhidro sulfuroso líquido, utilizando tecnología INCO, antes de su envío a la presa de residuos y su posterior recirculación como A.P. a la planta, (Fig. 1).

Los análisis de los metales comunes del agua, en partes por millón, arrojaron los siguientes valores: Zn: 0,10; Cd: 0,04; Co: 0,20; Na: 600; K: 26; Ca: 65; $\mathrm{Ni}: 0,00 ; \mathrm{Cu}: 80$.

El cobre presente formaba parte de complejos cianurados cuprosos solubles, generados durante el proceso de cianuración. La presencia de uno u otra especie: $\left[\mathrm{Cu}(\mathrm{CN})_{2}\right]^{-},\left[\mathrm{Cu}(\mathrm{CN})_{3}\right]^{2-}$ y $\left[\mathrm{Cu}(\mathrm{CN})_{4}\right]^{3-}$,

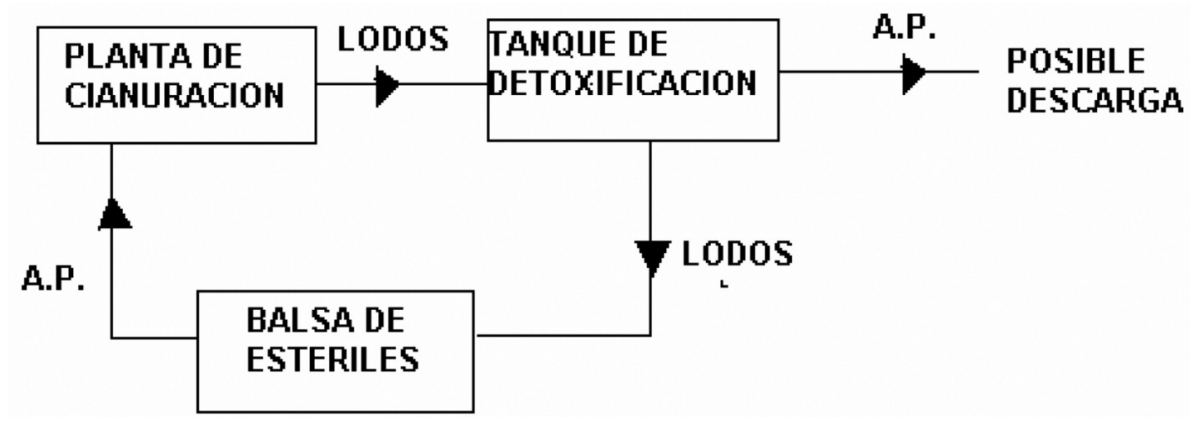

Figura 1. Esquema de circuito de detoxificación.

Figure 1. Schematic diagram of a detoxification circuit. 
depende del $\mathrm{pH}$, de la temperatura, así como de la concentración total de cobre y cianuro presente ${ }^{[17]}$. Dadas las condiciones de lixiviación llevadas a cabo en la planta "El Valle" ( $\mathrm{pH}$ elevado y altas concentraciones de cianuro) $)^{[17-23]}$, el cobre en solución se encuentra principalmente en forma de $\left[\mathrm{Cu}(\mathrm{CN})_{3}\right]^{2-}$, aunque por reacciones de disociación se pueden producir los otros complejos descritos más arriba.

\subsection{Permanganato Potásico}

El permanganato potásico es un fuerte oxidante ampliamente utilizado para el tratamiento de aguas y que, como ya se ha señalado anteriormente, estudios previos han demostrado que se comporta como un eficaz detoxificante de aguas cianuradas. Para lograr una reacción de oxidación eficaz y segura del cianuro, ésta debe de realizarse en condiciones fuertemente alcalinas (pH: 12-14) [24], lo que se consigue añadiendo las cantidades necesarias de $\mathrm{Ca}(\mathrm{OH})_{2}$ en concentraciones superiores a $1,8 \mathrm{~g} / \mathrm{l}$, según la reacción (2).

$$
\begin{aligned}
& 2 \mathrm{MnO}_{4}^{-}+3 \mathrm{CN}^{-}+\mathrm{H}_{2} \mathrm{O} \rightarrow \\
& 2 \mathrm{MnO}_{2}+3 \mathrm{CON}^{-}+2 \mathrm{OH}^{-}
\end{aligned}
$$

En el caso de este trabajo, el reactivo empleado se recibió en forma sólida, en bidones de $25 \mathrm{~kg}$ provenientes de la empresa Carus Nalón (TrubiaAsturias) y se disolvió en tanques de 1.0001 para conseguir una mezcla al 2,5\% que permite su utilización sin tener el problema de una posible cristalización del reactivo, pues su solubilidad depende de la temperatura ${ }^{[25]}$.

\subsection{Cal}

Para trabajar dentro de los límites de seguridad exigidos por el proceso se utilizó lechada de cal para alcanzar los niveles de alcalinidad necesarios ${ }^{[25-29]}$. Esta lechada procedía de una derivación de la propia planta metalúrgica de tratamiento industrial que la utilizaba como regulador de $\mathrm{pH}$, precipitante $\mathrm{y}$ acondicionante del medio. El reactivo se alimentaba por la parte superior de la tolva.

\subsection{Métodos de medida}

Como método de medida para control de los resultados se utilizó un espectrofotómetro Brickman para la determinación del cianuro por el método del ácido pícrico y espectrofotometría de absorción atómica para el $\mathrm{Cu}$.

Por agilidad del proceso, dado que la determinación del cianuro se podía obtener directamente en el laboratorio instalado a pie de planta para este estudio, se realizaron sólo controles sobre la evolución de este parámetro y, muy puntualmente, sobre el cobre para corroborar que las relaciones de eliminación de ambos componentes se mantenían.

\section{DISEÑO Y CONSTRUCCIÓN}

La planta piloto diseñada, cómo se observa en la figura 2, constaba de un tanque de $5 \mathrm{~m}^{3}$ de capacidad con un agitador interior formado por dos hélices a distintas alturas conectado a un motor con variador de frecuencia

La alimentación del agua a tratar al tanque se realizó mediante una tolva-embudo superior que descargaba a la mitad de la altura del tanque, logrando de este modo, una mezcla más homogénea.

Dentro del tanque se instaló un electrodo para medición del pH, cuya señal procesada por un PLC, permitía controlar la dosificación de la lechada de cal y mantener de este modo la alcalinidad en el valor deseado.

Paralela a la tubería de la lechada se instaló otra para la dosificación del permanganato potásico líquido, disuelto al 2,5 \% como se ha señalado anteriormente, en la misma tolva mencionada. El reactivo era bombeado desde el tanque depósito mediante una bomba de membrana de teflón con accionamiento eléctrico. El volumen de permanganato requerido se controlaba de manera manual, subiendo o bajando el caudal de la bomba dosificadora dependiendo de los análisis de cianuro que se realizaban cada hora para controlar la calidad del agua de entrada.

\section{ENSAYOS Y DISCUSIÓN DE LOS RESULTADOS}

La puesta en funcionamiento de la planta se realizaba a primera hora de la mañana llenando el tanque mezclador y analizando las condiciones iniciales del agua a tratar, dejándola en funcionamiento todo el día de manera continua.

El tiempo de permanencia del agua de proceso en el tanque mezclador se calculó en función del caudal de agua de entrada (aproximadamente 1,39 1/s), de la capacidad del tanque y de la velocidad de ascensión del agua hasta el rebose, resultando ser una hora. 


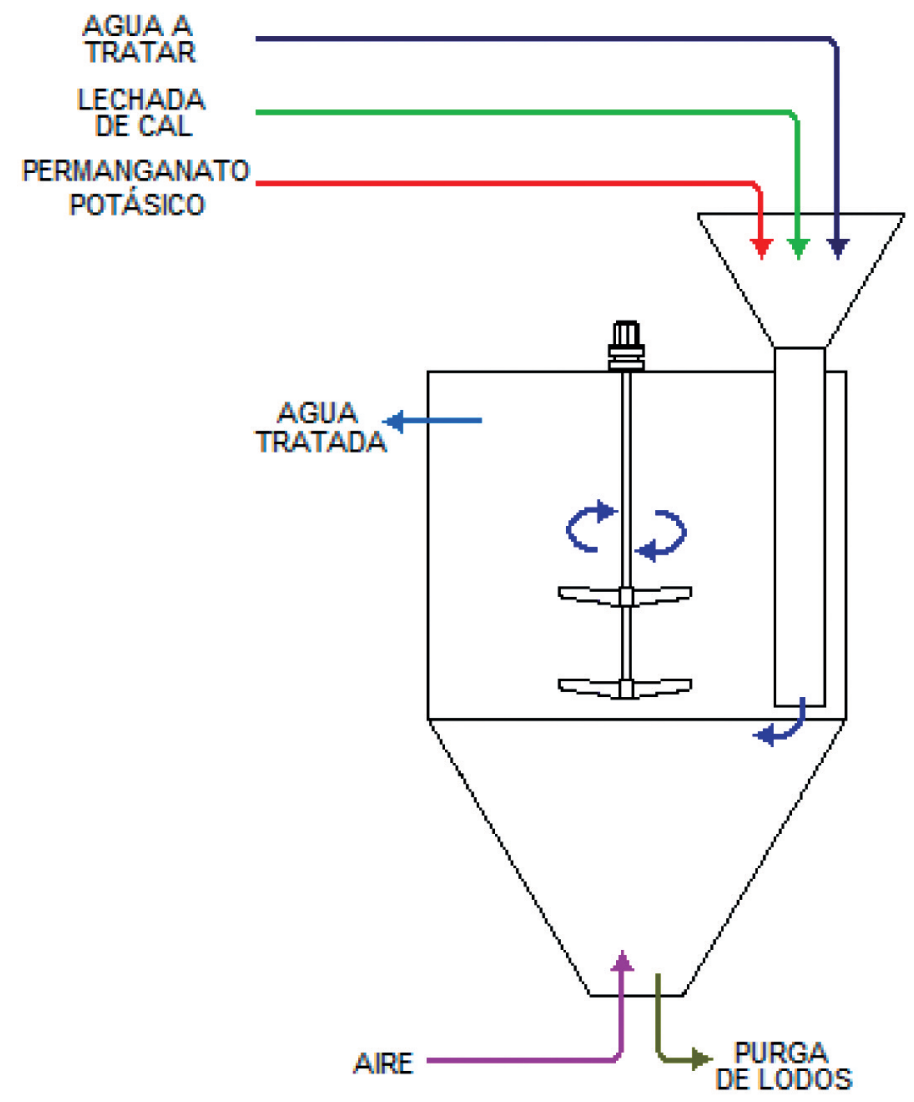

Figura 2. Esquema general de la planta piloto.

Figure 2. General scheme of the pilot plant.

Las muestras para análisis, tanto del agua de proceso como a la salida del tanque mezclador, se recogían a dicho intervalo de tiempo.

El agua una vez tratada salía por un rebosadero situado en la parte superior del tanque. Se evacuaba mediante una canaleta hasta una pequeña "balsa" donde los precipitados sólidos, formados por hidróxido de cobre y dióxido de manganeso principalmente, acababan depositándose.

Una vez conocido el caudal de entrada del agua de proceso en el tanque, su concentración inicial en cianuro y puesto en funcionamiento el PLC de control de la lechada de cal para ajustar el $\mathrm{pH}$ a las condiciones de trabajo, la técnica de trabajo consistió en comenzar los ensayos con una baja dosificación de permanganato en relación a la inicial de cianuro, para ir aumentando la proporción de dicho reactivo en días sucesivos. La agitación del tanque se fijó en $1.500 \mathrm{rpm}$.

La mayor dificultad operativa radicó en el control manual de la bomba de alimentación para la dosificación del permanganato, ya que al ser un proceso en continuo en el que las condiciones del agua varían frecuentemente, no era fácil obtener un control exacto del reactivo añadido.
Aunque de los estudios previos en laboratorio se deduce que el porcentaje de detoxificación aumenta a medida que lo hace el reactivo añadido, alcanzándose su valor óptimo para una relación en peso de permanganato a cianuro de 5 a 1 , en una primera fase de la experimentación a escala industrial se trabajó con porcentajes en peso inferiores a 3 para comprobar si el trabajo en un sistema con mejor hidrodinámica, podría mejorar el aprovechamiento del reactivo. Posteriormente, se fue aumentando la dosificación empleada hacia los valores encontrados en el laboratorio. Las tablas I y II presentan los primeros resultados de detoxificación obtenidos en días consecutivos y que alcanzan valores del $50 \%$ al final del día.

Continuando con el esquema planteado para los ensayos, en las tablas III y IV se recogen las pruebas realizadas en días consecutivos, con una mayor dosificación de permanganato manteniendo constantes el resto de los parámetros, siendo la única variable las condiciones iniciales del agua de proceso. Estos resultados ponen de manifiesto que la detoxificación se encuentra en el entorno del $50 \%$ para todas las adiciones, por lo que la concentración final del 
Tabla I. Resultados del tratamiento del A.P. (agua de proceso) con permanganato potásico en la planta piloto con baja dosificación de permanganato. Ensayo 1

Table I. Treatment of A.P. (water process) with potassium permanganate in the pilot plant with low doses of permanganate. Test I

\begin{tabular}{|c|c|c|c|c|c|c|}
\hline \multirow{2}{*}{ ADICIONES } & \multicolumn{2}{|c|}{ ppm CN- } & \multicolumn{3}{|c|}{$\mathrm{KMnO}_{4}$} & \multirow{2}{*}{$\begin{array}{c}\mathrm{KMnO}_{4} \mathrm{I} \\
\mathrm{CN}^{-} \text {(peso) }\end{array}$} \\
\hline & ENT. & SAL. & $\%$ detox & $\mathrm{ml} / \mathrm{min}$ & $\mathrm{kg} / \mathrm{h}$ & \\
\hline 1 & 50,0 & & & 98 & 0,15 & 0,6 \\
\hline 2 & 52,6 & 36,0 & 28,0 & 100 & 0,15 & 0,6 \\
\hline 3 & 51,5 & 37,0 & 29,7 & 98 & 0,15 & 0,6 \\
\hline 4 & 50,2 & 38,1 & 26,0 & 116 & 0,17 & 0,7 \\
\hline 5 & 52,3 & 34,4 & 31,4 & 252 & 0,38 & 1,4 \\
\hline 6 & 50,2 & 35,1 & 33,0 & 236 & 0,35 & 1,4 \\
\hline 7 & 50,5 & 34,4 & 31,6 & 235 & 0,35 & 1,4 \\
\hline 8 & 51,4 & 32,0 & 36,6 & 242 & 0,36 & 1,4 \\
\hline 9 & 53,6 & 35,0 & 31,8 & 264 & 0,40 & 1,5 \\
\hline 10 & 53,0 & 31,0 & 42,1 & 430 & 0,65 & 2,5 \\
\hline 11 & 52,4 & 26,0 & 51,5 & 432 & 0,65 & 2,5 \\
\hline
\end{tabular}

Tabla II. Resultados del tratamiento del A.P. (agua de proceso) con permanganato potásico en la planta piloto con baja dosificación de permanganato. Ensayo II

Table II. Treatment of A.P. (water process) with potassium permanganate in the pilot plant with low doses of permanganate. Test II

\begin{tabular}{|c|c|c|c|c|c|c|}
\hline \multirow{2}{*}{ ADICIONES } & \multicolumn{3}{|c|}{ ppm CN- } & \multicolumn{2}{|c|}{$\mathrm{KMnO}_{4}$} & \multirow{2}{*}{$\begin{array}{c}\mathrm{KMnO}_{4} \mathrm{I} \\
\mathrm{CN}^{-} \text {(peso) }\end{array}$} \\
\hline & ENT. & SAL. & $\%$ detox & $\mathrm{ml} / \mathrm{min}$ & $\mathrm{kg} / \mathrm{h}$ & \\
\hline 1 & 49,6 & & & 100 & 0,15 & 0,6 \\
\hline 2 & 52,6 & 36,0 & 27,4 & 100 & 0,15 & 0,6 \\
\hline 3 & 51,5 & 37,0 & 29,7 & 98 & 0,15 & 0,6 \\
\hline 4 & 47,0 & 36,1 & 30,0 & 86 & 0,13 & 0,6 \\
\hline 5 & 46,2 & 34,3 & 27,0 & 112 & 0,17 & 0,9 \\
\hline 6 & 50,2 & 32,1 & 30,6 & 236 & 0,35 & 1,4 \\
\hline 7 & 50,0 & 34,3 & 31,7 & 242 & 0,36 & 1,7 \\
\hline 8 & 50,0 & 31,8 & 38,3 & 252 & 0,38 & 1,7 \\
\hline 9 & 53,0 & 25,0 & 46,8 & 430 & 0,65 & 2,5 \\
\hline
\end{tabular}

cianuro en las aguas es muy superior a la exigida para el envío a cauce público.

La bibliografía consultada así como por los resultados previos obtenidos en el laboratorio y publicados en un artículo anterior ${ }^{[16]}$, han permitido calcular que la dosificación óptima de permanganato para alcanzar los niveles de detoxificación deseados en relación al cianuro inicial en el agua a tratar es de 5 a 1 (con excesos sobre la estequiometría para $\mathrm{CN}^{-}$ puro del orden del $70 \%$ ), lo que se ha podido com- probar. En la tabla $\mathrm{V}$ los resultados de los ensayos realizados con dosificaciones de permanganato potásico entre 4 y 6 , permiten alcanzar resultados de eliminación en torno al $95 \%$ consiguiendo de este modo el objetivo inicial buscado: obtener una concentración de cianuro en el agua que permite el envío a cauce público. Las trazas de cianuro residual que persisten en el agua, durante el proceso de traslado hasta su destino final, son destruidas por acción del propio aire o de la luz solar. 
APLICACIÓN DEL PERMANGANATO POTÁSICO PARA LA ELIMINACIÓN DE CIANUROS DE COBRE EN AGUAS RESIDUALES DE LA PLANTA DE LIXIVIACIÓN EN UNA MINA DE ORO (II): ENSAYOS EN PLANTA PILOTO USES OF THE POTASSIUM PERMANGANATE TO ELIMINATE COPPER CYANIDE FROM WASTE WATER RESULTING FROM A LIXIVIATION PLANT IN A GOLD MINE (II): PLLOT PLANT EXPERIENCES

Tabla III. Resultados del tratamiento del A.P. (agua de proceso) con permanganato potásico en la planta piloto con valores medios de dosificación de permanganato. Ensayo I

Table III. Treatment of A.P. (water process) with potassium permanganate in the pilot plant with a medium dosage of permanganate. Test I

\begin{tabular}{|c|c|c|c|c|c|c|}
\hline \multirow{2}{*}{ ADICIONES } & \multicolumn{3}{|c|}{ ppm $\mathrm{CN}^{-}$} & \multicolumn{2}{|c|}{$\mathrm{KMnO}_{4}$} & \multirow{2}{*}{$\begin{array}{c}\mathrm{KMnO}_{4} / \\
\mathrm{CN}^{-} \text {(peso) }\end{array}$} \\
\hline & ENT. & SAL. & $\%$ detox & $\mathrm{ml} / \mathrm{min}$ & $\mathrm{kg} / \mathrm{h}$ & \\
\hline 1 & 51,0 & & & 162 & 0,24 & 1,9 \\
\hline 2 & 51,5 & 28,6 & 44,4 & 162 & 0,24 & 1,9 \\
\hline 3 & 54,9 & 26,0 & 50,0 & 228 & 0,34 & 2,7 \\
\hline 4 & 54,9 & 26,4 & 48,8 & 282 & 0,42 & 3,1 \\
\hline 5 & 52,1 & 25,2 & 54,0 & 282 & 0,42 & 3,1 \\
\hline 6 & 51,5 & 24,4 & 52,3 & 300 & 0,45 & 3,5 \\
\hline
\end{tabular}

Tabla IV. Resultados del tratamiento del A.P. (agua de proceso) con permanganato potásico en la planta piloto con valores medios de dosificación de permanganato. Ensayo II

Table IV. Treatment of A.P. (water process) with potassium permanganate in the pilot plant with a medium dosage of permanganate. Test II

\begin{tabular}{|c|c|c|c|c|c|c|}
\hline \multirow{2}{*}{ ADICIONES } & \multicolumn{3}{|c|}{ ppm $\mathrm{CN}^{-}$} & \multicolumn{2}{|c|}{$\mathrm{KMnO}_{4}$} & \multirow{2}{*}{$\begin{array}{c}\mathrm{KMnO}_{4} \mathrm{I} \\
\mathrm{CN}^{-} \text {(peso) }\end{array}$} \\
\hline & ENT. & SAL. & $\%$ detox & $\mathrm{ml} / \mathrm{min}$ & $\mathrm{kg} / \mathrm{h}$ & \\
\hline 1 & 52,4 & & & 220 & 0,33 & 2,5 \\
\hline 2 & 52,4 & 28,6 & 45,4 & 220 & 0,33 & 2,5 \\
\hline 3 & 51,3 & 25,3 & 51,7 & 228 & 0,34 & 2,7 \\
\hline 4 & 51,5 & 28,6 & 44,1 & 228 & 0,34 & 2,7 \\
\hline 5 & 50,1 & 28,1 & 46,2 & 228 & 0,34 & 2,7 \\
\hline 6 & 52,0 & 25,2 & 49,7 & 228 & 0,34 & 2,7 \\
\hline 7 & 54,9 & 26.0 & 50,0 & 228 & 0,34 & 2,7 \\
\hline 8 & 52,3 & 26,5 & 51,7 & 240 & 0,36 & 2,8 \\
\hline 9 & 54,9 & 24,4 & 53,4 & 282 & 0,42 & 3,1 \\
\hline 10 & 52,3 & 25,0 & 51,4 & 300 & 0,45 & 3,4 \\
\hline
\end{tabular}

A la vista de los buenos resultados obtenidos para el cianuro, en los ensayos siguientes se analiza también la evolución que experimenta el cobre. La tabla VI refleja los resultados obtenidos en condiciones similares a las de la tabla anterior obteniéndose para el caso del cobre eliminaciones superiores al $95 \%$.

\subsection{Modificaciones al método}

Una vez verificada la eficacia oxidativa del permanganato potásico y la viabilidad del método en continuo, se pasó a realizar una serie de modificaciones en algunos de los parámetros que intervienen en el proceso, con el fin de intentar una mejora del tratamiento.

\subsubsection{Velocidad de Agitación}

En la tabla VII se observan resultados con distintas velocidades de agitación. Aunque se mantienen constantes tanto el caudal del agua de entrada como la dosificación de permanganato potásico, las variaciones en la contaminación inicial del agua de proceso implican cambios en la relación final existente entre el reactivo añadido y el cianuro inicial. 
Tabla V. Resultados del tratamiento del A.P.(agua de proceso) con permanganato potásico en la planta piloto con dosificaciones elevadas. Ensayo I

Table V. Treatment of AP (process water) with potassium permanganate in the pilot plant with a high dosage of potassium permanganate. Test I

\begin{tabular}{ccccccc}
\hline \multirow{2}{*}{ ADICIONES } & \multicolumn{3}{c}{ ppm CN } & \multicolumn{2}{c}{$\mathbf{K M n O}_{\mathbf{4}}$} & \multicolumn{1}{c}{$\begin{array}{c}\mathbf{K M n O}_{\mathbf{4}} \mathbf{I} \\
\mathbf{C N}^{-} \text {(peso) }\end{array}$} \\
\cline { 2 - 6 } & ENT. & SAL. & \% detox & ml/min & kg/h & \\
\hline 1 & 50,7 & & & 750 & 1,13 & 4,1 \\
2 & 53,1 & 2,2 & 95,8 & 800 & 1,20 & 4,1 \\
3 & 50,8 & 2,2 & 95,8 & 800 & 1,20 & 4,4 \\
4 & 50,0 & 6,7 & 86,8 & 800 & 1,20 & 4,4 \\
5 & 52,4 & 4,5 & 91,2 & 920 & 1,38 & 5,1 \\
6 & 52,4 & 2,0 & 96,1 & 920 & 1,50 & 5,1 \\
7 & 51,2 & 3,6 & 93,2 & 920 & 1,38 & 5,2 \\
8 & 50,0 & 2,8 & 94,4 & 1000 & 1,50 & 5,6 \\
9 & 46,0 & 2,4 & 95,2 & 1000 & 1,50 & 5,8 \\
10 & 51,6 & 2,6 & 94,4 & 1100 & 1,65 & 5,9 \\
\hline
\end{tabular}

Tabla VI. Resultados del tratamiento del A.P.(agua de proceso) con permanganato potásico en la planta piloto con alta dosificación de permanganato. Ensayo II

Table VI. Treatment of AP (process water) with potassium permanganate in the pilot plant with a high dosage of potassium permanganate. Test II

\begin{tabular}{|c|c|c|c|c|c|c|c|c|}
\hline \multirow{2}{*}{ ADICIONES } & \multicolumn{3}{|c|}{ ppm $\mathrm{CN}^{-}$} & \multicolumn{3}{|c|}{ ppm Cu } & \multirow{2}{*}{$\underset{\mathrm{kg} / \mathrm{h}}{\mathrm{KMnO}_{4}}$} & \multirow{2}{*}{$\begin{array}{c}\mathrm{KMnO}_{4} / \mathrm{CN}^{-} \\
\text {(peso) }\end{array}$} \\
\hline & ENT. & SAL. & $\%$ detox & ENT. & SAL. & $\%$ detox & & \\
\hline 1 & 40,4 & & & 60,0 & & & 1,78 & 5,50 \\
\hline 2 & 38,4 & 4,1 & 90,0 & 63,8 & 3,0 & 95,0 & 1,78 & 5,79 \\
\hline 3 & 38,2 & 2,3 & 94,0 & 60,2 & 2,6 & 95,7 & 1,78 & 5,83 \\
\hline 4 & 37,7 & 3,2 & 91.8 & 60,7 & 2,3 & 96,4 & 1,78 & 5,90 \\
\hline 5 & 35,7 & 2,2 & 94,2 & 59,3 & 1,9 & 96,9 & 1,78 & 6,24 \\
\hline
\end{tabular}

A pesar de esta pequeña diferencia en la relación de permanganato a cianuro, es evidente una mejoría en los resultados de los ensayos realizados con una agitación muy fuerte. Este hecho es debido al aumento del oxígeno en solución provocado por la agitación violenta favoreciendo, por tanto, la destrucción del complejo cianurado.

Otros ensayos de agitación y aumento de oxígeno se realizaron mediante la colocación de una válvula en la parte inferior del tanque que permitía soplar aire a presión ( $900 \mathrm{dm}^{3} \mathrm{~N}$ por minuto) a la mezcla, con la agitación fijada en $1.500 \mathrm{rpm}$. Los resultados de la tabla VIII corresponden a dichos ensayos, en los que se puede ver que la detoxificación aumenta todavía más, alcanzando niveles cercanos al $99 \%$ de eliminación para el cobre y del $95 \%$ para el cianuro.

\subsection{Cálculo aproximado del coste del proceso}

Dada la simplicidad de la planta no se ha calculado el costo de la misma por ser realmente bajo y asumible la construcción de los tanques, dosificadores y los equipos controladores del proceso. Por ello, se ha centrado el estudio económico en el coste de funcionamiento, en particular del más importante, que son los reactivos.

Para ello, se parte de una concentración inicial media del cianuro en solución de 40 ppm. Como ya se ha señalado, por los datos obtenidos tanto a nivel de laboratorio como a nivel de planta piloto, los resultados aconsejan la utilización de una relación en peso 
APLICACIÓN DEL PERMANGANATO POTÁSICO PARA LA ELIMINACIÓN DE CIANUROS DE COBRE EN AGUAS RESIDUALES DE LA PLANTA DE LIXIVIACIÓN EN UNA MINA DE ORO (II): ENSAYOS EN PLANTA PILOTO USES OF THE POTASSIUM PERMANGANATE TO ELIMINATE COPPER CYANIDE FROM WASTE WATER RESULTING FROM A LIXIVIATION PLANT IN A GOLD MINE (II): PLLOT PLANT EXPERIENCES

Tabla VII. Resultados del tratamiento del A.P. con permanganato potásico en la planta piloto con variación en la velocidad de agitación

Table VII. Treatment of A.P. with potassium permanganate in the pilot plant varying the stirring speed

\begin{tabular}{|c|c|c|c|c|c|c|c|c|c|}
\hline \multirow{2}{*}{ ADICIONES } & \multirow{2}{*}{$\begin{array}{c}\text { Agitac. } \\
\text { (rpm) }\end{array}$} & \multicolumn{3}{|c|}{ ppm CN${ }^{-}$} & \multicolumn{3}{|c|}{ ppm Cu } & \multirow{2}{*}{$\begin{array}{c}\mathrm{KMnO}_{4} \\
\mathrm{~kg} / \mathrm{h}\end{array}$} & \multirow{2}{*}{$\begin{array}{c}\mathrm{KMnO}_{4} \mathrm{I} \\
\mathrm{CN}^{-} \text {(peso) }\end{array}$} \\
\hline & & ENT. & SAL. & $\%$ detox & ENT. & SAL. & $\%$ detox & & \\
\hline 1 & 1.500 & 40,44 & & & 63,8 & & & 1,78 & 5,50 \\
\hline 2 & 1.500 & 38,395 & 4,1 & 90,0 & 60,7 & 2,3 & 96,4 & 1,78 & 5,79 \\
\hline 3 & 1.500 & 38,42 & 3,1 & 91,8 & 59,6 & 2,3 & 96,3 & 1,78 & 5,80 \\
\hline 4 & 2.347 & 38,20 & 2,3 & 94,0 & 60,2 & 2,6 & 95,6 & 1,78 & 5,83 \\
\hline 5 & 2.347 & 38,18 & 2,3 & 94,1 & 59,3 & 1,9 & 96,8 & 1,78 & 5,83 \\
\hline 6 & 2.347 & 38,383 & 2,2 & 94,1 & 60,0 & 1,0 & 98,3 & 1,78 & 5,87 \\
\hline
\end{tabular}

Tabla.VIII. Resultados del tratamiento del A.P.(agua de proceso) con permanganato potásico en la planta piloto con aire soplado por la parte inferior del tanque

TablaVIII. Treatment of A.P. (water process) with potassium permanganate in the pilot plant with air blown by the bottom of the tank

\begin{tabular}{|c|c|c|c|c|c|c|c|c|}
\hline \multirow{2}{*}{ ADICIONES } & \multicolumn{3}{|c|}{ ppm CN- } & \multicolumn{3}{|c|}{ ppm Cu } & \multirow{2}{*}{$\underset{\mathrm{Kg} / \mathrm{h}}{\mathrm{KMnO}_{4}}$} & \multirow{2}{*}{$\begin{array}{c}\mathrm{KMnO}_{4} / \mathrm{CN}^{-} \\
(\text {peso) }\end{array}$} \\
\hline & ENT. & SAL. & $\%$ detox & ENT. & SAL. & $\%$ detox & & \\
\hline 1 & 33,5 & & & 63,9 & & & 1,54 & 5,75 \\
\hline 2 & 33,5 & 2,1 & 93,6 & 63,9 & 0,8 & 98,8 & 1,54 & 5,75 \\
\hline 3 & 32,6 & 2,1 & 93,8 & 65,2 & 1,1 & 98,3 & 1,54 & 5,92 \\
\hline 4 & 34,4 & 2,0 & 93,8 & 62,8 & 1,0 & 98,5 & 1,54 & 5,59 \\
\hline 5 & 32,4 & 2,3 & 93,5 & 61,5 & 1.3 & 97,9 & 1,54 & 5,95 \\
\hline 6 & 32,4 & 2,0 & 93,7 & 62,7 & 1,3 & 97,9 & 1,54 & 5,95 \\
\hline
\end{tabular}

de $\mathrm{KMnO}_{4}$ a $\mathrm{CN}^{-}$de 5 a 1 , por lo que serían necesarios $8 \mathrm{ml}$ de disolución de $\mathrm{KMnO}_{4}$ al 2,5\% por litro de agua contaminada.

Suponiendo un caudal a tratar de $10.000 \mathrm{l} / \mathrm{h}$, se necesitarían unos 801 de $\mathrm{KMnO}_{4} /$ hora. Como con $1 \mathrm{~kg}$ de $\mathrm{KMnO}_{4}$ puro se obtienen 40 litros de solución de $\mathrm{KMnO}_{4}$ al 2,5 \% y el precio aproximado ofrecido por la empresa Brenntang en el año 2009 fue de $4 € / \mathrm{kg}$ para barriles de $25 \mathrm{~kg}$, se obtiene un precio de $0,1 € /$ litro de la solución. Por tanto se tendría un gasto diario de $192 € /$ día.

Por otra parte, son necesarios $1,8 \mathrm{~g} / \mathrm{l}$ de cal para alcanzar el $\mathrm{pH}$ de trabajo. El precio ofrecido por la misma empresa Brenntang fue de 0,24€/kg, por lo que serían necesarios 103,68 €/día, si bien las experiencias de la planta piloto han puesto de manifiesto cómo en un trabajo en continuo, este consumo es mucho menor, por lo que el coste también se reduciría.
Suponiendo un gasto energético más mantenimiento de $50 €$ y $100 €$ los gastos de operación y amortización, se obtendría un coste total de $445.68 €$ /día, para conseguir la detoxificación del agua en las condiciones anteriormente señaladas, valor muy interesante frente a los costes de procesos de detoxificación actuales, dado que la planta que operase en estas condiciones, puede facturar entre 100.000 y $200.000 €$ /día a un precio del oro entre 400-800 €/onza (en la actualidad supera los $1.100 € /$ onza, pero se toma unos precios que pueden ser más normales fuera de periodos de crisis). Los costes anteriores se pueden resumir según la siguiente expresión:

$$
\begin{gathered}
\mathrm{C}_{\mathrm{T}}=\mathrm{C}_{\mathrm{E}+\mathrm{M}}+\mathrm{C}_{\mathrm{O}+\mathrm{A}}+\left(3 \mathrm{Q}_{\mathrm{T}}\right) \\
{\left[\left(\mathrm{C}_{\mathrm{Ci}} \times \mathrm{P}_{\mathrm{KMnO} 4} / 25.000\right)+\left(9 \mathrm{P}_{\mathrm{cal}} / 625\right)\right]}
\end{gathered}
$$




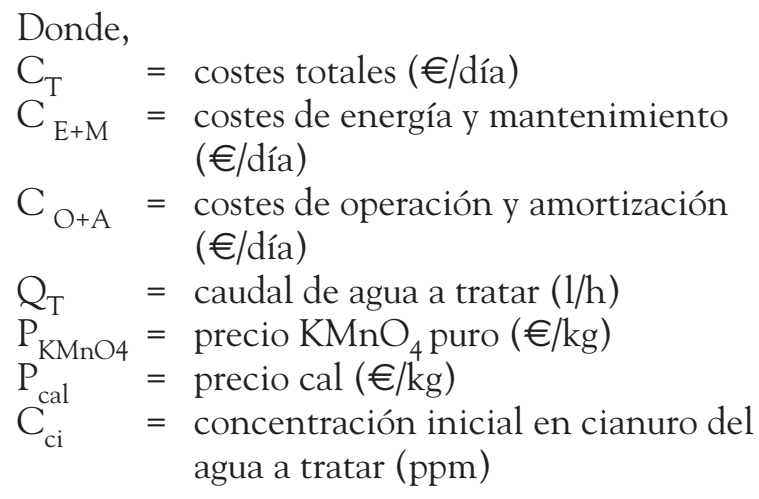

\section{CONCLUSIONES}

- El estudio realizado sobre la efectividad del método desarrollado en el laboratorio para el uso del permanganato potásico como oxidante de complejos cianurados, preferentemente de cobre, en aguas contaminadas de plantas hidrometalúrgicas, ha permitido obtener niveles altos de eliminación de este complejo, en continuo.

- A partir de relaciones de detoxificación en peso de permanganato a cianuro de 5 a 1 , se alcanzan reducciones superiores al $90 \%$ en cianuro y del $95 \%$ en cobre, lo que permite obtener un agua apta bien para recircular a planta o bien para envío a cauce público. En el caso del cianuro, las trazas residuales que persisten en el agua, son destruidas por acción del propio aire o de la luz solar durante el proceso de traslado hasta su destino final.

- Aplicando los resultados previos obtenidos en laboratorio a una instalación piloto, se demostró que es viable la aplicación del método en continuo, con un control automático de la dosificación de reactivos, manteniendo para ello un análisis permanente de los parámetros críticos del proceso, es decir, de la concentración inicial y final de cianuro en solución y del $\mathrm{pH}$. De este modo se pueden mantener las condiciones del proceso de oxidación dentro de un intervalo de valores óptimo para que tenga lugar.

- Los ensayos realizados han demostrado que el uso de una velocidad de agitación mayor o la inyección de aire a presión en el tanque de tratamiento, métodos ambos que favorecen la homogeneización de la mezcla inicial, así como una mayor incorporación de oxígeno a la solución, favorece el resultado final del tratamiento.

- Finalmente se puede concluir que el proceso ha sido patentado por ser un método novedoso, económico y eficaz ${ }^{[30]}$.

\section{Agradecimientos}

Los autores desean agradecer a la compañía Río Nacea Gold Mines, S.A. por financiar y facilitar la realización de este trabajo.

\section{REFERENCIAS}

[1] F. Habashi, C.I.M. Bulletin 80 (1987) 108-114.

[2] P. Navarro, C. Vargas, R. Alvarez y F.. Alguacil, Rev. Metal. Madrid 41 (2005) 12-20.

[3] P. Navarro, C. Vargas, FJ. Alguacil y R. Alvarez, Rev. Metal. Madrid, Vol. Extr. (2005) 69-73.

[4] J.J. Yáñez-Traslaviña, M.A. Vargas-Avila, I.H. García-Páez y J.E. Pedraza-Rosas, Rev. Metal. Madrid 41 (2005) 116-125.

[5] L. Chacón, F. Ruiz y R. Zapico, Rev. Metal. Madrid, Vol. Extr. (2005) 390-394.

[6] F. Nava-Alonso, E. Elorza-Rodríguez, A. UribeSalas y R. Pérez-Garibay, Rev. Metal. Madrid 43 (2007) 20-28.

[7] J.S. Scott y J. Ingles, Canadian Mineral Processors Thirteenth Annual Meeting, The Canadian Mineral Processors Society (Eds.), Otawa, Canadá, 1981, pp. 380-418.

[8] J.D. Desai, C. Ramakrishna, P.S. Patel y S.K. Awasthi, Chemical Engineering World XXXIII (6), EXICOM, India Pvt Ltd (Eds.), Mumbai, India,1998, pp. 115-121.

[9] Diario Oficial de las Comunidades Europeas, L 330, 5/12/1998, Directiva 98/83/CE del Consejo, 3/11/1998.

[10] B. Fernández, Tesis Doctoral, E.T.S.I.M.O., Universidad de Oviedo, 2007.

[11] R.R. Dash, A. Gaur y Ch. Balomajumder, J. Harzard. Mater. 163 (2009) 1-11.

[12] R.R. Dash, Ch. Balomajumder y A. Kumar. J. Harzard. Mater. 152 (2008) 387-396.

[13] T.I. Mudder y M. Botz, Eur. J. Miner. Process. Environ. Prot. 4 (2004) 100-110.

[14] J.P. Sancho, B. Fernandez y F. Bellón, $9^{\text {th }}$ Int. Mine Water Cong., J. Loredo \& F. Pendás (Eds.), Oviedo, España, 2005, pp. 413-417.

[15] A. Akcil, Biotechnol. Adv. 21 (2003) 501-511.

[16] J.P. Sancho, B. Fernández, J. Ayala, M.P. García y A. Lavandeira. Rev. Metal. Madrid 45 (2009) 415-423.

[17] A. Ballester, J. Sancho y L.F. Verdeja, Metalurgia Extractiva. Fundamentos, Ed. Síntesis, S.A., España, 2000, pp. 1-512.

[18] A.G. Sharpe, The Chemistry of Cyano Complexes of the Transition Metals, Ed. Academic Press, New York, EE. UU., 1976, pp. 1-300. 